\title{
Progress of Home Healthcare Sensor in Our Experience: Development of Wearable and Unobtrusive Monitoring
}

\author{
Toshiyo TAMURA*, \#
}

\begin{abstract}
Information technology has been applied to health management. In our laboratory, we have developed and produced prototypes of new sensors, and attempted to monitor various health parameters noninvasively and unobtrusively. This paper reviews the results of our research related to sensor development. The contents include unobtrusive monitoring in the bed, bath and toilet, and smart house. Then wearable sensors used for photoplethysmography, inertia sensor, deep body temperature measurement, and oxygen uptake monitor are presented. Finally, the requirement of regulatory science is commented
\end{abstract}

Keywords: unobtrusive monitoring, wearable monitoring, regulatory science.

Adv Biomed Eng. 9: pp. 189-196, 2020.

\section{Introduction}

Based on the request of the editorial office of the Japan Society for Medical and Biological Engineering to write a review of the author's research undertaken until now, this article looks back on the history of research on sensor development for the purpose of health management.

The research was triggered by the situation in the latter half of the 1980s, when aging of the population became an issue, raising concern over the increase of medical expenses in the future. In order to allow the population to live a healthy long life, approaches for disease prevention and disease prediction were proposed and initiated.

On the other hand, in 1988, the concept of ubiquitous computing was advocated, which was integration of computers into the society, such that the computer functions can be used any time, any place. Along with this development, we advocated ubiquitous health management, so that provides health information can be obtained any time, any place. After 20 years, the term "ubiquitous" has almost become obsolete, and more specific terms have been proposed, such as Internet of Things (IoT) and the Internet of Health Things (IoHT), which are systems that connect to the Internet for health management.

In Japan, the long-term care insurance system was started in April 2000, in preparation for the aging society.

Received on July 15, 2019; revised on September 2, 2019; accepted on September 2, 2019.

* Intitute of Healthcare Robotics, Future Robotics Organization, Waseda University

\#E-mail: t.tamura1949@gmail.com
Accompanying this initiative, subsidies for the purchase of information devices were approved, and equipment for the detection of fall from the bed and devices for prevention of wandering of dementia patients became more commonly used.

With this background, this article looks back on the history of the development of systems that we advocated, including unobtrusive measurement and management of health conditions as well as unconstrained or minimally constrained methods for obtaining biological information.

\section{Smart room and smart house using uncon- strained/unobtrusive biological measure- ments}

In the latter half of the 1980s, Dr. Tatsuo Togawa's Laboratory at the Tokyo Medical and Dental Institute of Tokyo Medical and Dental University initiated the concept of acquiring health information by simpler methods through obtaining biological information without attaching electrodes or sensors to the subjects, which was not possible with the conventional clinical test methods. This concept was also in consideration of how to monitor the health of elderly people in a super-aging society. We studied the feasibility of designing a method that does not require attaching electrodes or sensors to the subject, which can measure biological data without the subject being aware of it, while fully protecting the confidentiality of personal information.

Discussions were started on how biometric information could be obtained from activities of daily living, and whether it was possible to measure during bedtime, excretion, and bathing which is a special Japanese habit. 
We first considered the possible measurements at bedtime. Discussion suggested that a conductive non-woven fabric could be developed, and it would be possible to measure heart rate and respiratory rate. However, this idea was abandoned because similar development has already been started in other institutions. We next focused was on body temperature. We developed a temperature sensor that provides information on the sleeping state by measuring the temperature distribution in the subject's bed during sleep [1,2]. In this device, the sensors can be arranged in a matrix pattern or in a strip form under the mattress.

As shown in Fig. 1, we estimated the presence or absence of body movement obtained from the temperature distribution in the bed, and used this device to improve the sleeping environment [3-6]. We applied this method to pressure sore prevention by monitoring body movement [4], and also to sleep management for children who were not attending school. Those children got out of bed several time during night and thus they missed to control daily rhythm and resisted going to school in the morning. [2].

Furthermore, in the field of image processing, the heart and respiratory rates were estimated by calculating the optical flow from video images. Highly accurate detection was possible from slight movement of the futon of a sleeping subject [8-10].

Next, since many accidents occur while taking a bath, we tried to measure electrocardiogram (ECG) heart rate, and blood pressure as physiological activities while taking a bath. Although several theoretical studies had examined ECGs acquired in bathtub, we removed the noise at the site and verified the differences in signal characteristics and conductivity $[11,12]$. Regarding the methodology, electrodes were attached to the inside of the bathtub and bioelectric signals were acquired via the hot water. The Japanese bathing custom is quite unique, and for this reason, the above studies were hardly accept-

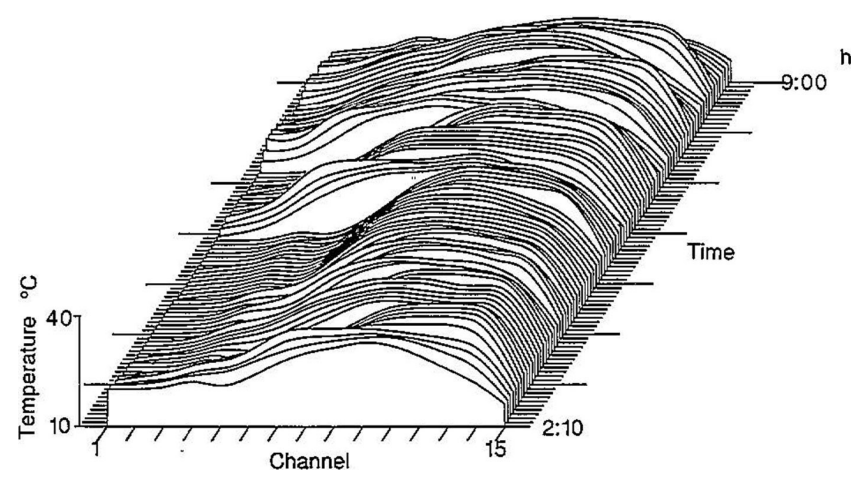

Fig. 1 Body movement from temperature distribution in the bed [5] with permission. ed at international academic community. Clinicians even commented that since the standard 12-lead ECG was medically the gold standard, our studies of ECG acquired in bathtub were not accurate. The reason was that because the electric signals were acquired through hot water, attenuation at low frequencies was large and the resulting waveform was completely different from that obtained on the standard 12-lead ECG. Eventually, current research is limited to detection of heart rate and heart rate variability. In recent years, some groups have tried to reproduce the conventional ECG by inverse problem analysis.

We set up a smart room in our laboratory that was equipped with the above-mentioned systems together with a precision weighing scale for detecting the amount of urine during urination, and attempted to acquire data [13]. During that study period, the acquired data were mainly processed with a computer, and were collected with different sampling rates and were displayed in an easy-to-understand manner. In addition, we attempted to identify individuals by personal recognition method when there were multiple users. Specifically, we attempted identification by performing wavelet analysis and neural network analysis using biological signals and ECGs. Although it was possible to distinguish between family members, while if there were 100 subjects, some subjects could not be distinguished.

We also proved that personal recognition was possible based on footprint, although it was necessary to take off the shoes [14].

In 1996, the New Energy and Technology Development Organization (NEDO) provided research sites that integrated the development of home care equipment and development of housing for elderly people. With the cooperation of local governments, Welfare Techno Houses were constructed in 15 locations nationwide. Although these houses were intended for research and development, a verification experiment was conducted in Takaoka, Toyama as a field site for unobtrusive measurement [15]. The experimental setup is shown in Fig. 2. In this experiment, we attempted to measure heart rate in bed using electroconductive fabric, heart rate during bathing, body weight at the toilet, and urine volume. Although a comparative study between young people and elderly people was attempted, continuous measurements over several days was not possible due to the purpose of facility utilization, and the experiment was terminated after a short period of time.

As an extension of the above experiment, we constructed a system that allowed estimation of lifestyle from the use frequencies of water, electricity and gas, instead of using medical equipment, and provided intervention when there was a possibility of illness $[16,17]$. 


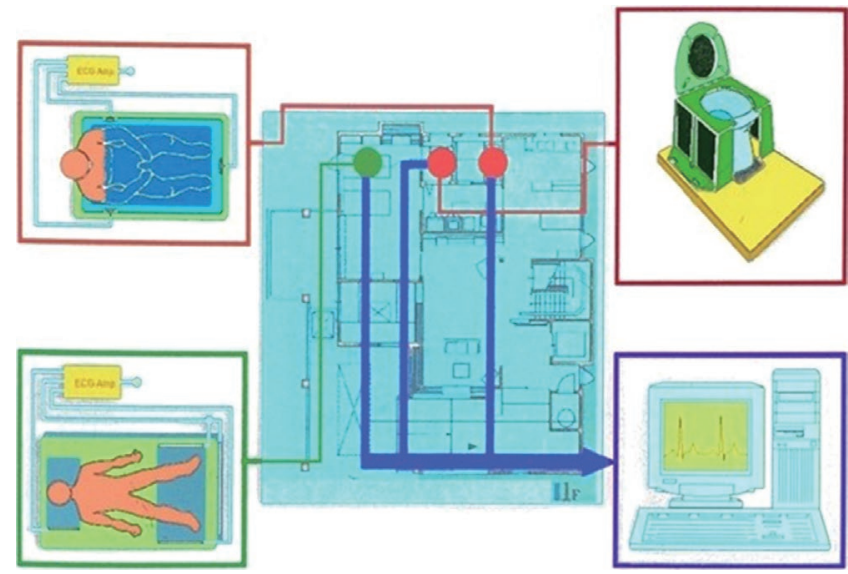

Fig. 2 Unobtrusive monitoring at smart house. The contents are ECG and heart rate at electro conductive sheet on the bed and bathtub ECG, and body weight and urinary volume at toilet [15].

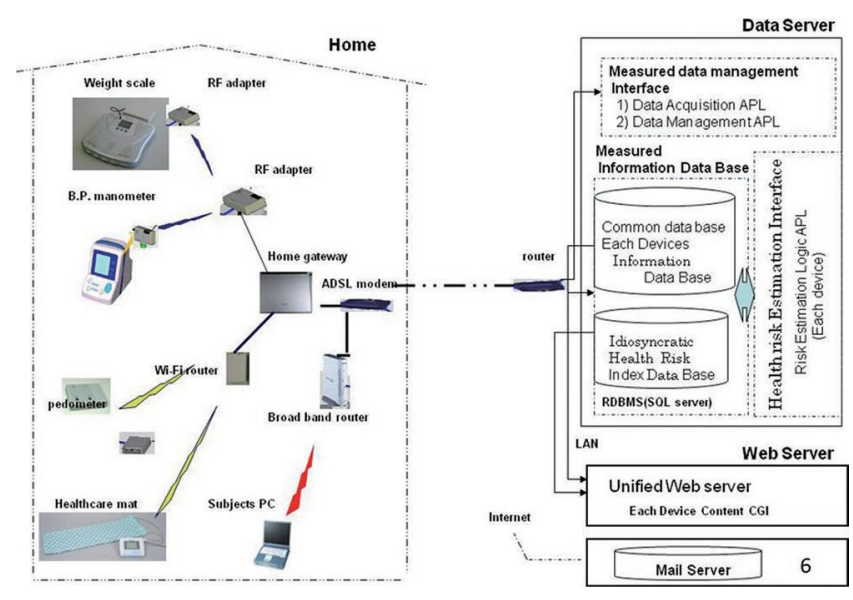

Fig. 3 An example of healthcare system [19] with permission.

Utilizing the habit of watching news on television in the elderly people's homes, the study suggested that it was possible to monitor the health condition from the history of using the remote control switch [17].

The use of wireless communication has become widespread, and remote management for home rehabilitation was also implemented [18].

In the first half of the 2000s, accompanying the increase in aged population which was unprecedented in industrial countries, there was an urgent need for assistive devices and building of home healthcare system to promote healthy longevity. Conceptually, the purpose was to effectively collect data using existing medical devices and use the data for health management [19,20]. An example is shown in Fig. 3.

From the concept of "any time, any place", we attempted to connect many biological information devices via the Internet and search for signs of illness from the data. However, there were problems with the reliability and validity of data acquisition, and the study was limited to construction of the system. Through this research and development, we created a standard connection protocol, which later became the prototype of the Continue specification that allows connection of health management devices from different manufacturers to computers and smart phones [21].

\section{Development of wearable photoplethysmog- raphy sensor and cuffless sphygmomanome- ter}

With the widespread use of the Internet, health management systems also became popular. Furthermore, from April 2008, specific health checkup and specific health guidance were started for people aged 40 to 74 years [22]. As a result, web-based healthcare systems that automatically collected data from household health management devices and chronologically analyzed the data to be predict and prevent disease.. The data focused on blood pressure, body weight and exercise, which were considered to be the most important health parameters. We analyzed the operational performance of these systems. The result of our study showed that even subjects who required specific health guidance monitored blood pressure for less than $10 \%$ of the time over a year [23]. Blood pressure is an important parameter, and it is important to measure continuously to know how the changes over time. We examined whether there was an alternative to the sphygmomanometer that used a cuff.

As one possibility, we considered the photoplethysmographic (PPG) method. Since its introduction by Hertzman in 1938, PPG has been widely used in the clinical setting for qualitative evaluation. The device consists of a light source, mainly LED, and a light receiving element, and can be manufactured easily. It mainly detects transmitted light. Conventionally, near-infrared light has been used, and the blood oxygen saturation is calculated from two wavelengths; red light and near-infrared light. In recent years, LEDs with strong light intensity have been developed with the improvement of semiconductor technology. Therefore, we focused on green light and produced the prototype of a reflective type green light PPG. Green light was not used until then because it is strongly absorbed by blood. However we found that the difference in absorption allowed clear detection of the difference in pulsation, and using reflected light gave a highly sensitive pulse waveform [24,25]. Moreover, our study showed that the green light was reflected from the relatively superficial layer of the skin, so that the influence of noise due to movement was small. However, during exercise, because of the larger influence of noise, 

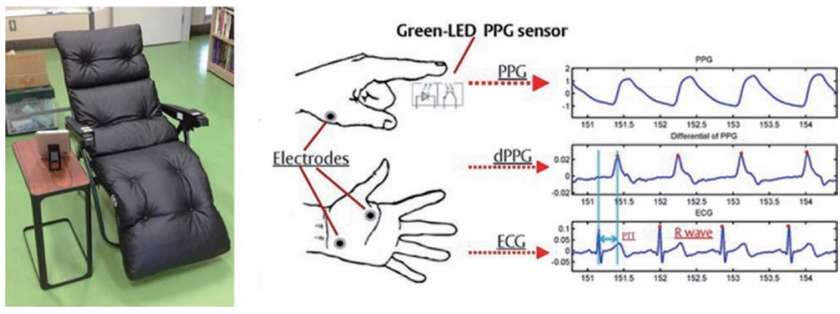

Fig. 4 Chair-based cuffless blood pressure monitor and the principle of detecting pulse transit time.

it was not always possible to obtain pulse waves with high accuracy. We have examined several major methods of noise processing. As a simple method, we have attempted to remove noise by simultaneously measuring motion with an accelerometer [26].

Furthermore, we were trying to estimate blood pressure as an application of the PPG method. The waveform detected on a PPG depicts information of the stiffness of blood vessels, and blood pressure can be estimated using a conversion formula. The general method of estimating blood pressure by PPG involves estimation from the original waveform and the second-derivative waveform of the PPG, and calculation of the pulse transit time from the ECG R wave and the PPG wave. In order to reduce the influence of noise, we acquired PPG and R wave of ECG in a sitting position on the chair and estimated the blood pressure. To estimate blood pressure by PPG, calibration with a cuff-type sphygmomanometer is required. Our device has been verified to have accuracy that complies with the ISO81060-2 clinical standards for cufftype sphygmomanometer [27].

\section{Verification of wearable inertia sensor and fall prevention}

At the same time as unconstrained measurement and unobtrusive measurement were being developed, we also attempted to develop devices using inertia sensor as wearable health management devices for the purpose of monitoring behavior when going out. Although it was the time before airbags in cars were widely used and accelerometers were expensive, there was a demand for quantification of gait in the field of welfare and rehabilitation. Our group started the development. One of the demands in the welfare field was to improve ADL of hemiplegic patients during walking [28] and to monitor the behaviors of patients with dementia [29]. By developing devices that are small and unobtrusive to the individuals being measured, we were able to contribute to research in the field of welfare and rehabilitation.

For health personnel in the clinical setting, utilization of accelerometer was often difficult to understand from the physical interpretation of acceleration (force $=$
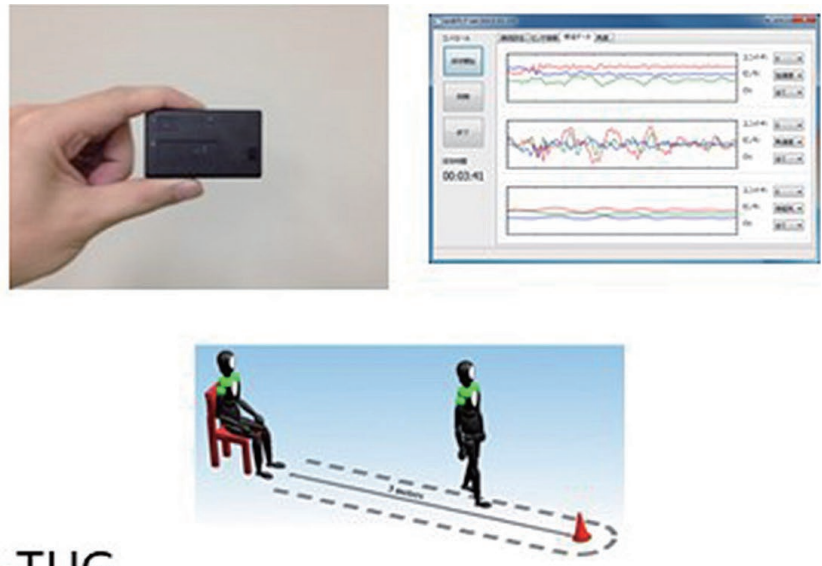

\section{i-TUG}

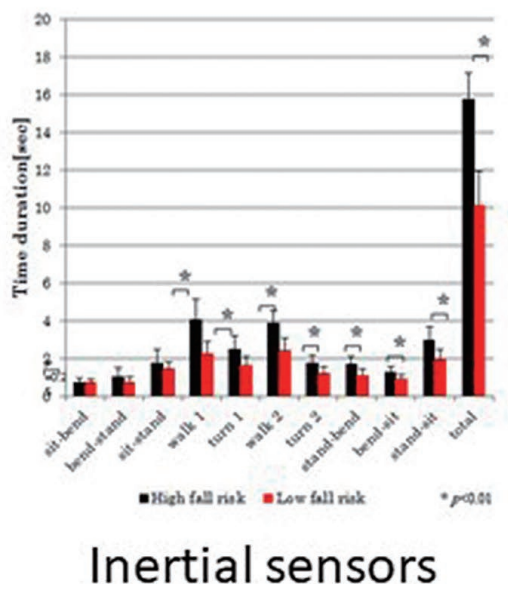

Fig. 5 Developed wearable accelerometer and its application for Time-up and Go test. Each phase such as sitting and walking can be detected and the time is differentiated by low and high fall risk subjects.

mass $\times$ acceleration), but it is now widely used for motion analysis and evaluation of the outcome of rehabilitation; for example, the evaluation of exercise load [30] and detailed evaluation of the timed up and go test [3133]. The timed up and go test is an ADL evaluation in rehabilitation, which comprehensively evaluates the complex movements from sitting on a chair to rising from the chair, walking, turning around, walking back to the chair, and sitting on the chair. By using an accelerometer, it has become possible to define the characteristics of each movement separately and provide suggestions as to which movement should be improved. Inertia sensor has been applied to detailed evaluation of posture $[34,35]$ (Fig. 5).

As a separate project, we attempted to classify gait patterns by utilizing a novel biological signal analysis method [36-41]. Although this method was mainly an application of wavelet analysis, we successfully classified ascending and descending stairs using this method.

Furthermore, with the increase in number of acci- 


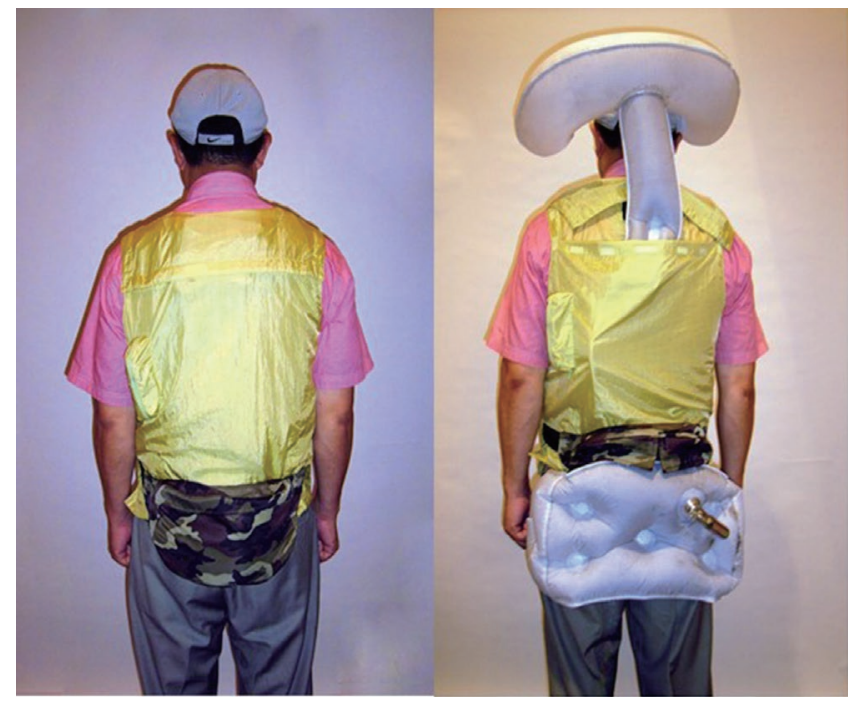

Fig. 6 Human airbag for prevention of fall by using accelerometer and angular velocity sensors. Left before fall Right after fall.

dents due to falls in the aging society, we have developed a device that predicts falls by analyzing the acceleration and angular velocity values at the time of falling, and inflates an airbag when the person falls, which prevents fractures, as shown in Fig. 6 [42]. The time until the airbag is inflated during a fall has to be within $200 \mathrm{~ms}$ from the detection of the fall trigger signal. Therefore, explosive was used to introduce gas into the airbag. Due to the explosive sound, its use in long-term care facilities was discouraged. However, it was applied to prevent accidents when falling from a high place and construction site.

\section{Development of deep body temperature ther- mometer based on dual-heat flux method}

The heat flux compensation method that measures the core temperature from the body surface is the main research theme of the Togawa Laboratory, and we have also developed temperature control of the body surface and the hypothermia method. In the heat flux compensation method, heat is applied from outside the body to apparently eliminate the heat flux from the inside of the body. The power is a problem for a portable device. Therefore, Professor Tetsu Nemoto of Kanazawa University proposed a method of estimating the deep body temperature by detecting the difference in heat flux. Based on our theoretical analysis and simulation experiments, we attempted to improve the accuracy of the device and developed a wearable device [43-45]. As shown in Fig. 7, assuming two heat fluxes, the deep body temperature is estimated from the temperature difference between sensors 1 and 2 and sensors 3 and 4. Estimation
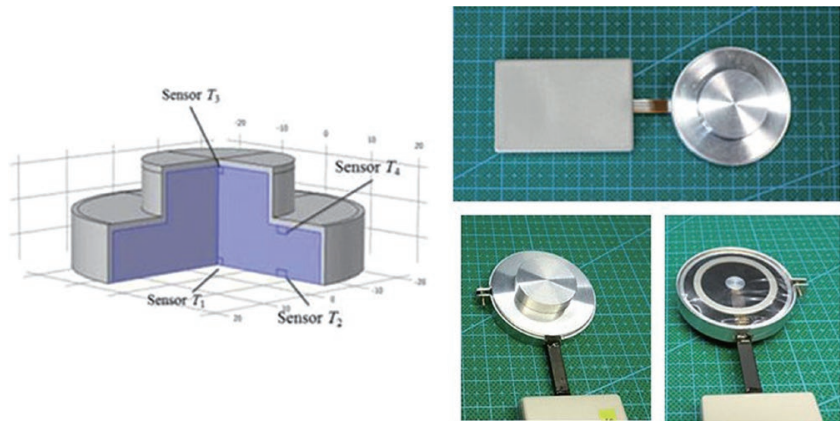

Fig. 7 Structure of dual-heat-fluxtype thermometer and photos of device with permission.

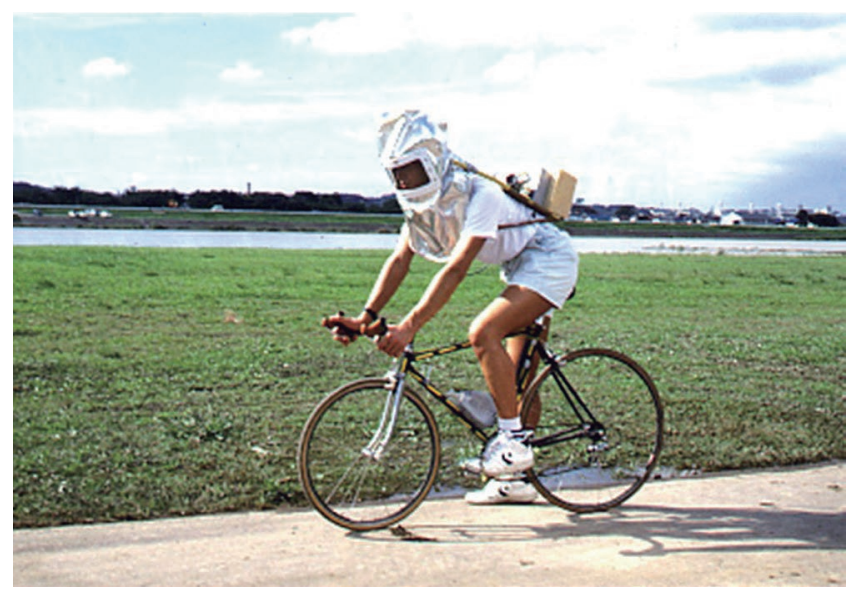

Fig. 8 Canopy-type wearable oxygen-uptake monitor.

of core temperature is an indicator for the prevention of heat stroke, and is expected to be applied to practical use in the future.

\section{Development of wearable oxygen uptake mea- surement system}

Indirect heat flux measurement is an important indicator for knowing the amount of energy metabolism. In general, for a portable oxygen uptake monitoring device, a subject wears a mask or a mouthpiece, and the oxygen concentration and carbon dioxide concentration in the exhaled gas as well as the ventilation volume are measured and used to calculate oxygen intake. Oxygen sensors such as small galvanic cell, Clark type electrode, and zirconia are used to measure oxygen concentration. Near-infrared light which reacts specifically with carbon dioxide gas concentration is commonly used to measure carbon dioxide concentration. The turbine type, pneumotach, and hot wire flowmeter are used to measure ventilation volume. We used a canopy (hood) to eliminate the hassle of wearing a mask. As shown in Fig. 8, in our method, the face is covered with a canopy, the gas containing a mixture of inhaled and exhaled gas is forcibly 
removed, and the gas concentrations at that time together with the ventilation volume (expiratory volume) are measured and used to calculate the oxygen intake [4648].

We attempted to reduce the size of the device, but we were only able to reduce it to approximately the size of a backpack. A galvanic sensor was used for sensing the oxygen concentration, but the battery capacity was large because of the need to forcibly and continuously suction the gas.

We also attempted to evaluate some commercially available devices, but that did not lead to the development of novel breakthrough devices. In recent years, there is renewed interest in the development of an oxygen uptake monitor that is simple and has acceptable error compared with the standard method using Doubly labeled water, for the study of obesity associated with lifestyle and nutritional management in elderly people [49].

\section{Regulatory science}

We have developed several devices so far, and approval by regulatory authorities is required for practical application and commercialization of these devices. In Japan, the Pharmaceuticals and Medical Devices Agency (PMDA) plays that role. If anyone wants to market a medical device, certification by a third-party certifying body or approval by PMDA is necessary depending on the type of medical device. PMDA approval is required if the product involves new principles or has the risk of cause serious harm that threatens life. Therefore, it is necessary to show evidence that the device is useful for medical treatment, especially to show the efficacy when used by doctors.

On the other hand, for medical devices, there are standards that specify accuracy, reliability, reproducibility, and measurement methods. These standards are voluntary, and specify things and matters that, if left uncontrolled, would become diversified, complicated, and disordered. Internationally, these certifying bodies include International Organization for Standardization (ISO), the International Electrotechnical Commission (IEC), and domestically, the Japanese Industrial Standards (JIS). Medical devices, such as automated sphygmomanometer, are specified in JIST 1115. The cuffless sphygmomanometer that is currently under development is not included in this category, and must be approved by PMDA before the product can be launched on the market. Considering the process and time to approval, creating a new standard is one approach. However, in order to create a standard, the social impact must be considered.

Even if the cuffless sphygmomanometer is used as a healthcare device, it will be dealt with as a medical de- vice. Therefore, there are high hurdles related to standards and approval as a medical device.

\section{Conclusion}

Looking back on the research over nearly half a century, we have summarized our research contents in several publications [50-60]. Health management via the Internet is being widely used, and a connected health management system as a service to customers has been advocated, which collects and analyzes the data obtained from sensor development. However, even if big data is collected, due to the nature of the living body, there are still many issues to be solved such as verification of sensor reliability and reproducibility as well as the necessity of preprocessing of the collected data. Although the research trend is to do noise analysis by processing the signals collected, development of sensors with high signalto-noise ratio should be continued. By 2025, the Japanese society will become one in which one elderly person will be supported by two or less young persons. There is an urgent need to create a society with healthy longevity by making full use of unobtrusive measurements and wearable devices. The roles of biomedical engineers are more important than ever before. I hope that everyone will play an active role.

\section{Conflicts of interest}

The authors declare no conflicts of interest with any companies or commercial organizations per the definition of Japanese Society for Medical and Biological Engineering.

\section{Acknowledgments}

The author gratefully acknowledge the guidance and advice kindly given by Professor Tatsuo Togawa, Professor Akira Kamiya, and the late Professor Kiichi Tsuchiya (Waseda University); and the contributions from the undergraduate students, graduate students, and staff of Waseda University, Tokyo Denki University, Keio University, Yamaguchi University, National Center for Geriatrics and Gerontology, Chiba University, Osaka ElectroCommunication University, and Nara Institute of Science and Technology.

Many of the studies were supported by the Ministry of Education, Culture, Sports, Science and Technology, the Ministry of Health, Labor and Welfare, the Ministry of Internal Affairs and Communications, the Ministry of Economy, Trade and Industry, and many private research foundations.

Research in biomedical engineering is conducted in close associations with doctors and medical personnel. The author expresses his thanks to Dr. Toshiro Fujimoto, former director of Fujimoto Hospital, Miyakonojo City, 
Miyazaki Prefecture.

Grants were received from JSPS Grants-in-Aid for Scientific Research JP18K01440 and JP18K12148, and AMED Research Grant JP18dk0310077 for writing of this paper.

\section{References}

1. Tamura T, Togawa T: Design of a multichannel solid-state recorder and its application to temperature measurement. Med Biol Eng Comput. 22, 411-147, 1984.

2. Tamura T, Togawa T, Murata M: A bed temperature monitoring system for assessing body movement during sleep. Clin Phys Meas. 9, 139-145, 1988.

3. Togawa T, Mizukami H, Tamura T: Physiological monitoring techniques for home health care. Biomed Sci Instrum. 28, 105$110,1992$.

4. Tamura T, Zhou J-X, Togawa T: Temperature monitoring for prevention of pressure sores. Sens Actuators. A21-23, 660-662, 1990.

5. Tamura T, Zhou J, Mizukaki H, Togawa T: A system for monitoring temperature distribution in bed and its application to the assessment of body movement. Physiol Meas. 14, 33-41, 1993.

6. Lu L, Tamura T, Togawa T: Detection of body movements during sleep by monitoring of bed temperature. Physiol Meas. 20, 137148, 1999.

7. Tamura T, Miyasako S, Ogawa M, Togawa T, Fujimoto T: Assessment of bed temperature monitoring for detecting body movement during sleep: comparison with simultaneous video image recording and actigraphy. J Med Eng Phys. 21(1), 1-8, 1999.

8. Nakajima K, Tamura T, Miike H: Monitoring of heart and respiratory rates by photoplethysmography using a digital filtering technique. Med Eng Phys. 18(5), 365-372, 1996.

9. Nakajima K, Osa A, Kasaoka S, Nakashima K, Maekawa T, Tamura T, Miike H: Detection of physiological parameters without any physical constraints in bed using sequential image processing. Jpn J Phys. 35, L269-272, 1996.

10. Nakajima K, Matsumoto Y, Tamura T: Development of real-time image sequence analysis for evaluating posture change and respiratory rate of the subject in bed. Physiol Meas. 22, N21-28, 2001.

11. Tamura T, Yoshimura T, Nakajima K, Miike H, Togawa T: Unconstrained heart rate monitoring during bathing. Biomed Instrum Tech. 31, 391-396, 1997.

12. Ogawa M, Tamura T, Toawa T: Automated Acquisition System for Routine, Noninvasive Monitoring of Physiological Data. Telemed J. 4(2), 177-185, 1998.

13. Tamura T, Togawa T, Ogawa M, Yoda M: Fully automated health monitoring system in the home. J Med Eng Phys. 20, 573-579, 1998.

14. Nakajima K, Mizukami Y, Tanaka K, Tamura T: Footprint-Based Personal Recognition. IEEE Trans Biomed Eng. 47(11), 15341537, 2000.

15. Tamura T, Kawarada A, Nambu M, Tsukada A, Sasaki K, Yamakoshi K: E-Healthcare at an Experimental Welfare Techno House in Japan. Open Med Inf J. 1, 1-7, 2007.

16. Tsukamoto S, Hoshino H, Tamura T: Easily Installable Wireless Behavioral Monitoring System with Electric Field Sensor for Or- dinary Houses. Open Med Inf J. 2, 49-57, 2008.

17. Nambu M, Nakajima K, Noshiro M, Tamura T: Technological developments in Japan - An algorithm for the automatic detection of health conditions - An image processing technique for diagnosing poor health in the elderly. IEEE Eng Med Biol Mag. 24(4), 38-42, 2005.

18. Masuda Y, Sekimoto M, Nambu M, Higashi Y, Fujimoto T, Chihara K, Tamura T: Technological developments in Japan - An unconstrained monitoring system for home rehabilitation - A wireless heart/respiratory rate sensor accessible to home-visit therapists. IEEE Eng Med Biol. 24(4), 32-37, 2005.

19. Tamura T, Mizukura I, Sekine M, Kimura Y: Monitoring and evaluation of blood pressure changes with a home healthcare system. IEEE Trans Inf Technol Biomed. 15(7), 602-607, 2011.

20. Nakajima K, Nambu M, Kiryu T, Tamura T, Sasaki K: Low-cost, email-based system for self-blood pressure monitoring at home. J Telemed Telecare. 12(4), 03-220, 2006.

21. Mizukura I, Tamura T, Kimura Y, Yu W: New Application of IEEE 11073 to Home Health Care. Open Med Inf J. 3, 44-53, 2009.

22. Tamura T, Kimura Y: Specific health checkups in Japan: The present situation analyzed using 5-year statistics and the future. Biomed Eng Lett. 5(1), 22-28, 2015.

23. Tamura T, Maeno S, Hattori T, Kimura Y, Kimura Y, Yoshida M, Minato K: Assessment of participation compliance with a webbased home healthcare system for promotion specific health checkups. Biocybernetics Biomed Eng. 34 (1), 63-69, 2014.

24. Maeda Y, Sekine M, Tamura T: The advantages of wearable green reflected photoplethysmography. J Med Syst. 35(5), 829-834, 2012.

25. Maeda Y, Sekine M, Tamura T: Relationship between measurement site and motion artifacts in wearable reflected photoplethysmography. J Med Syst. 35(5), 969-976, 2012.

26. Tamura T, Maeda Y, Sekine M, Yoshida M: Wearable photoplethysmographic sensors - past and present. Electronics. 3, 281-302, 2014.

27. Tang Z, Sekine M, Tamura T, Tanaka N, Yoshida M, Chen W: Measurement and estimation of 3D orientation using magnetic and inertial sensors. Adv Biomed Eng. 4, 135-143, 2015.

28. Fujimoto T, Shimooki S, Tamura T, Togawa T: Measurement of physical movement in normal subjects and patients with hemiplegia by a portable acceleration monitoring system. $J$ Ambulatory Monit. 9(2), 149-162, 1996.

29. Tamura T, Fujimoto T, Sakaki H, Higashi Y, Yoshida T, Togawa T: A solid-state ambulatory physical activity monitor and its application to measuring daily activity of the elderly. J Med Eng Tech. 21, 96-105, 1997.

30. Tamura T, Nakajima K, Togawa T, Wakabayashi R, Osano M: Development of a new exercise test for children. Med Biol Eng Comput. 23, 482-486, 1985.

31. Higashi Y, Yamakoshi K, Fujimoto T, Sekine M, Tamura T: Quantitative evaluation of movement using the timed up-and-go test. IEEE Eng Med Biol Mag. 27(4), 38-46, 2008.

32. Zakaria NA, Kuwae Y, Tamura T, Minato K, Kanaya S: Quantitative analysis of fall risk using TUG test. Comput Methods Biomech Biomed Eng. 2013. doi: 10.1080/10255842.2013.805211

33. Tanaka N, Zakaria NA, Kibinge NK, Kanaya S, Tamura T, Yoshida M: Fall-risk classification of the timed up-and-go test with 
principle component analysis. Int J Neurorehabil Eng. 1, 106, 2014.

34. Martinez-Mendez R, Sekine M, Tamura T: Detection of anticipatory postural adjustments prior to gait initiation using inertial wearable sensors. Comput Methods Biomech Biomed Eng. 2011. doi: $10.1080 / 10255842.2011 .565753$

35. Martinez-Mendez R, Sekine M, Tamura T: Detection of anticipatory postural adjustments prior to gait initiation using inertial wearable sensors. J Neuro Eng Rehabil. 8(17), 1186-1743, 2011.

36. Tamura T, Sekine M, Ogawa M, Togawa T, Fukii Y: Classification of acceleration waveforms during walking by Wavelet transform. Meth Inf Med. 36, 356-359, 1997.

37. Sekine M, Tamura T, Togawa T, Fukui Y: Classification of waist-acceleration signals in a continuous walking record. Med Eng Phys. 22, 285-291, 2000.

38. Sekine M, Tamura T, Akay M, Togawa T, Fukui Y: Analysis of acceleration signals in healthy young subjects using Wavelet transform method. Method Inf Med. 39, 183-185, 2000.

39. Sekine M, Tamura T, Akay M, Fujimoto T, Togawa T, Fukui Y: Discrimination of walking patterns using wavelet-based fractal analysis. IEEE Trans Neural Syst Rehabil Eng. 10, 188-196, 2002.

40. Akay M, Sekine M, Tamura T, Higashi Y, Fujimoto T: Unconstrained monitoring of body motion during walking. IEEE Eng Med Biol Mag. 22(3), 104-109,2003.

41. Sekine M, Tamura T, Yoshida M, Suda Y, Kimura Y, Miyoshi H, Kijima Y, Higashi Y, Fujimoto T: A gait abnormality measure based on root mean square of trunk acceleration. J Neuro Eng Rehabil. 10, 118, 2013.

42. Tamura T, Yoshimura T, Sekine M, Uchida M, Tanaka O: A wearable airbag to prevent fall injuries. IEEE Trans ITB. 13 (6), 910914, 2009.

43. Huang M, Tamura T, Tang Z, Chen W, Kanaya S: A wearable thermometry for core body temperature measurement. IEEE J Biomed Health Inf. doi: 10.1109/JBHI.2016.2532933

44. Huang M, Tamura T, Tang Z, Chen W, Kanaya S: Structural optimization of a wearable deep body thermometer: from theoretical simulation to experimental verification. J Sens. 2016, 2016. doi: dx.doi.org/10.1155/2016/4828093

45. Huang M, Tamura T, Chen W, Kanaya S: Evaluation of structural and thermophysical effects on the measurement accuracy of deep body thermometers based on dual-heat-flux method. J Therm Biol. 47, 26-31, 2015.

46. Tamura T, Nemoto T, Nakajima K, Togawa T: Portable device for monitoring oxygen uptake. Med BioI Eng Comput. 24, 186-192, 1986.

47. Tamura T, Higuchi N, Sato K, Togawa T: Evaluation of ambulatory oxygen uptake measurement system. In: Hermans GPH ed. Sports, Medicine and Health, Elsevier Science Ltd, pp. 10041009, 1990.

48. Tamura T, Sato, K, Togawa T: Ambulatory oxygen uptake monitor. IEEE Trans Biomed Eng. 39, 1274-1282, 1992.

49. Ichinoseki-Sekine N, Yoshimura T, Nambu M, Tamura T: Evaluation of oxygen uptake monitors in low flow volumes using a respiratory simulator. J Clin Eng. 30, 145-152, 2005.

50. Tamura T: Body motion analysis. In: Öberg PÅ, Togawa T, Spelman FA ed. Sensors in Medicine and Health Care, Wiley VCH, pp. 243-281, 2004.
51. Tamura T: Home health care devices. In: Webster JG ed. Encyclopedia of Medical Devices and Instrumentation, Second Edition, Vol. 3, Wiley Interscience, pp. 525-536, 2006.

52. Tamura T: Sensing Technologies for Biomedical Telemetry. In: Nikita KS ed. Handbook of Biomedical Telemetry, IEEE Wiley, pp. 76-130, 2014.

53. Tamura T: Blood flow. In: Brahme A ed. Comprehensive biomedical Physics, Elsevier, 2014.

54. Tamura T: Wearable inertial sensors and their applications. In: Sazonov E and Neuman R eds. Wearable Sensors, Elsevier, 2014.

55. Tamura T: Topical review Home geriatric physiological measurement. Physiol Meas. 33, R47-65, 2012.

56. Tamura T, Chen W: Seamless monitoring of physiological information in daily life. Adv Biomed Eng. 4, pp.86-95, 2015.

57. Tamura T, Chen W (eds): Seamless Healthcare Monitoring. Springer, 2018.

58. Tamura T, Huang M, Togawa T. Current developments in wearable thermometer. Adv Biomed Eng. 7, 88-99, 2018.

59. Tamura T: Current progress of photoplethysmography and $\mathrm{SPO}_{2}$ for health monitoring. Biomed Eng Lett. 9(1), 21-36, 2019.

60. Tamura T: Wearable oxygen uptake and energy monitors. Physiol Meas. in press.

\section{Toshiyo Tamura}

Dr. Toshiyo TAmuRa received his Ph.D. from Tokyo Medical and Dental University in 1980. He is currently a visiting professor, Future Robotics Organization, Waseda University, Japan. His research interests include biomedical instrumentation, biosignal processing, telemedicine telecare, home

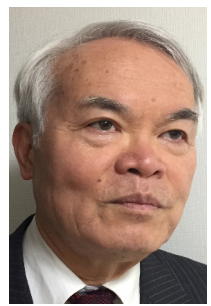
care technology and rehabilitation engineering. His research has resulted in over 300 English reviewed articles. His and his colleagues'book entitled "Biomedical sensors and instruments" and "Seamless healthcare monitoring" are popular textbooks for bioinstrumentation and medical devices. He also wrote several chapters including sensors for telemedicine and application of wearable inertia sensors. He is involved regional innovation strategy support program and creates innovations for achieving healthy and long life through the development of health care systems with smart bioinstrumentation and testing. He has developed unobtrusive monitoring systems and wearable devices for improving the quality of life. He has served as a chair of IEEE/EMBS Tokyo Chapter in 1996-2000, and the Asian Pacific representative for the EMBS from 2000 to 2004. He is fellows of IAMBE, Japanese Society of Medical Electronics and Biological Engineering, Japanese Society of Life Support Technology, and Japanese Society for Nursing Science and Engineering. 